Mémoires du livre

Studies in Book Culture

\title{
L'éditeur de littérature consacrée face au chercheur en sciences sociales
} Dits, non-dits et contrepoints heuristiques

\section{Lilas Bass}

Volume 10, numéro 2, printemps 2019

Les discours de l'éditeur

The Publisher's Discourse

URI : https://id.erudit.org/iderudit/1060970ar

DOI : https://doi.org/10.7202/1060970ar

Aller au sommaire du numéro

Éditeur(s)

Groupe de recherches et d'études sur le livre au Québec

ISSN

1920-602X (numérique)

Découvrir la revue

Citer cet article

Bass, L. (2019). L'éditeur de littérature consacrée face au chercheur en sciences sociales : dits, non-dits et contrepoints heuristiques. Mémoires du livre / Studies in Book Culture, 10(2). https://doi.org/10.7202/1060970ar

\section{Résumé de l'article}

Cet article propose de rendre compte des discours des éditeurs littéraires français s'inscrivant au sein du pôle de l'édition consacrée et de les confronter à des outils d'analyse issus principalement de la sociologie, de l'ethnographie et de l'histoire pour saisir au mieux la manière dont ces éditeurs se donnent à voir face au chercheur en sciences sociales. Il s'agira donc, d'une part, de déterminer ce qui gouverne les discours des éditeurs appartenant au pôle de l'édition consacrée, en fonction du degré de consécration de chaque éditeur au sein du pôle, et, d'autre part, de proposer des outils méthodologiques (observation, discours des gatekepeers, comptage et recours aux archives) permettant de mettre au jour les stratégies de domination éditoriale qui assurent à ces éditeurs une légitimité particulière dans le champ éditorial français, voire international.

Tous droits réservés ( C Groupe de recherches et d'études sur le livre au Québec, 2019 


\title{
Mémoires du livre Studies in Book Culture
}

\section{L'ÉDITEUR DE LITTÉRATURE CONSACRÉE FACE AU CHERCHEUR EN SCIENCES SOCIALES : \\ Dits, non-dits et contrepoints heuristiques}

\author{
Lilas BASS \\ École des Hautes Études en Sciences Sociales
}

Cet article propose de rendre compte des discours des éditeurs littéraires français s'inscrivant au sein du pôle de l'édition consacrée et de les confronter à des outils d'analyse issus principalement de la sociologie, de l'ethnographie et de l'histoire pour saisir au mieux la manière dont ces éditeurs se donnent à voir face au chercheur en sciences sociales. Il s'agira donc, d'une part, de déterminer ce qui gouverne les discours des éditeurs appartenant au pôle de l'édition consacrée, en fonction du degré de consécration de chaque éditeur au sein du pôle, et, d'autre part, de proposer des outils méthodologiques (observation, discours des gatekepeers, comptage et recours aux archives) permettant de mettre au jour les stratégies de domination éditoriale qui assurent à ces éditeurs une légitimité particulière dans le champ éditorial français, voire international.

This article sets out to examine the discourses of French publishers within the field of sanctioned publishing. Analytical tools derived primarily from sociology, ethnography and history are applied in order to better understand ways in which these publishers position themselves in relation to research in the social sciences. On the one hand, the article determines what governs the discourses of publishers working in the field of sanctioned literature in terms of each publisher's degree of dedication to the field, and on the other hand, proposes methodological tools (observation, speeches by gatekeepers, counting, and archival research) which reveal publishing domination strategies that ensure these editors a particular legitimacy in the French and even international publishing fields. 
Entamer une recherche sur l'édition littéraire parisienne consacrée n'est pas tâche aisée pour le·la chercheur·se. Cet article propose de revenir sur les discours des éditeurs littéraires français s'inscrivant au sein du pôle de l'édition consacrée et de les confronter à des outils d'analyse issus principalement de la sociologie, de l'ethnographie et de l'histoire, afin de saisir au mieux la manière dont ces éditeurs se donnent à voir face au·à la chercheur·se en sciences sociales.

Comme dans tous les milieux d'« entre-soi », et à plus forte raison d' « élite ${ }^{1}$ ", dont les membres ont des caractéristiques sociales analogues et dont les réseaux sont très exclusifs, les modalités d'accès sont à négocier perpétuellement et à ajuster tout au long de la recherche. Pour autant, cette difficulté d'accès à un terrain relativement fermé permet au·à la chercheur·se, armé e d'outils d'objectivation, de cerner, au gré des ajustements successifs à l'imposition d'hexis verbales et corporelles ${ }^{2}$ - pour partie pensées, au risque de « faux pas $^{3} »$, pour partie inconsciemment acquises au fil d'une socialisation de terrain et qui échappent malheureusement à l'analyse -, les cadres de pensée qui «parlent » à l'éditeur. Aussi les refus, les écueils et les maladresses sont-ils autant de matériaux précieux à l'analyse pour saisir en creux la manière dont l'éditeur comprend ou non son environnement social - et donc la manière dont il se pense et se présente.

Dans le même temps, et au-delà des "ratés", notamment des refus d'entretiens ${ }^{4}$, des refus d'enregistrement ou de la perte de contrôle au cours d'un entretien avec un «imposant ${ }^{5}$ », le la sociologue a à sa disposition des outils permettant d'objectiver les discours que l'éditeur tient sur lui-même, son catalogue et son métier.

Plus spécifiquement, l'étude thématisée des discours donne accès à la manière dont éditeur se présente, ne laissant entrevoir qu'une partie de son travail et de sa position objective dans le champ littéraire. La mobilisation d'outils issus de l'ethnographie, tels que le comptage ${ }^{6}$, l'observation ethnographique ${ }^{7}$ et le discours des gatekeepers, ou issus de la tradition historienne comme le traitement des archives, permet de proposer des contrepoints heuristiques pour mettre en perspective les discours de l'éditeur et révéler au mieux ce qu'il dit, mais surtout ce qu'il ne dit pas. Ainsi, l'ensemble des dits et des non-dits 
offre un meilleur portrait des enjeux de « représentation ${ }^{8}$ » qui risqueraient, sans ces contrepoints, d'être invisibilisés par le seul recours aux entretiens.

En d'autres termes, de quelles manières et à quelles conditions le la sociologue peut-il accéder aux éditeurs français du pôle consacré de l'édition littéraire? Que révèlent ces conditions d'accès de la " posture $^{9}$ » tenue par l'ensemble des éditeurs étudiés et de la posture tenue par chaque éditeur en fonction de sa situation dans le «champ littéraire ${ }^{10} 》$ ? Enfin, comment réussir à s'affranchir des discours souvent fort maitrisés par des acteurs conscients de la nécessité de contrôler leur histoire et leur rôle, et, par-là, parvenir à objectiver les stratégies de domination éditoriale?

Ce travail proposera de concilier une approche en termes de « champ » et une approche compréhensive - la confrontation de deux écoles (beckerienne ${ }^{11}$ et bourdieusienne ${ }^{12}$ ) et le croisement d'outils méthodologiques s'avérant particulièrement heuristiques dans le cadre d'un terrain difficile à appréhender ${ }^{13}$. La combinaison des outils développés par l'interactionnisme symbolique, d'une part, et la sociologie des champs, d'autre part, permettra en effet de mettre en lumière un acteur - l'éditeur appartenant au pôle de l'édition consacrée - pris dans des logiques de distinction et de légitimation en même temps que nécessairement soumis à l'activité collective - bien que celle-ci soit, comme nous le verrons, souvent déniée. Ce déni d'activité collective nous renseigne d'ailleurs sur la posture récurrente tenue par les éditeurs du sous-champ étudié, celle d'un individu qui bâtit seul son activité et son identité en ne faisant pas mention des deux aspects révélés par les principaux courants de la sociologie de la culture : déni de l'économie d'une part ${ }^{14}$; déni du travail collectif d'autre part. L'éditeur existe en tant que figure publique dans cet espace qu'il construit et se donne à voir comme personnage unique et singulier d'une production littéraire «désintéressée ${ }^{15}$ ». Mise à l'épreuve du comptage de son catalogue, de l'ethnographie ou encore des discours des gatekeepers, cette posture, qui lève le soupçon de cupidité d'une part et le distingue du commun des « personnels de renfort ${ }^{16} »$ de la littérature d'autre part, permet de replacer l'éditeur dans « une mosaïque de "marchésréseaux ${ }^{17 "}$ " plus ou moins cloisonnés ${ }^{18} »$, de relations interpersonnelles, voire « interpersonnalisées». Par ailleurs, cette double approche permettra de rendre compte d'éditeurs conscients de la nécessité de maîtriser leur propre histoire pour légitimer la valeur de leur métier et, par-là, des productions qu'ils 
imposent sur le marché des « biens symboliques ${ }^{19}$ »-à plus forte raison dans un contexte de rationalisation et de concentration éditoriale qui contraint l'éditeur, depuis les années 1980, à tenir d'autant mieux sa posture d'homme double ${ }^{20}$.

En effet, le «sous-champ de production restreinte ${ }^{21}$ » étudié s'est avéré réticent à l'objectivation, pour des raisons intrinsèques à son mode d'accès et de fonctionnement, qui le rend par ailleurs cohérent dans son ensemble. Il s'agira ici de penser les discours des éditeurs appartenant au pôle de l'édition littéraire consacrée, à savoir « ceux qui dominent le champ de production et le marché par le capital économique et symbolique qu'ils ont su accumuler ${ }^{22}$ ». Les maisons d'édition étudiées trouvent ainsi leur place du côté du pôle de l'édition consacrée en ce que leurs auteur'e's concourent et remportent régulièrement des prix littéraires (notamment les grands prix d'automne) et en ce que ces maisons contraignent les « nouveaux entrants ${ }^{23}$ » dans le champ à se positionner en fonction des propositions et des prises de position littéraires de ce pôle. Par ailleurs, ce pôle de l'édition consacrée apparait d'une grande homogénéité lorsque l'on considère les caractéristiques sociales des membres qui le composent : ces derniers sont en effet majoritairement des hommes (à l'exception d'un tandem homme-femme au sein de la maison la moins consacrée du pôle étudié); blancs; vivant à Paris; ayant entre 42 et 64 ans au moment de l'étude; très diplômés (bac +5 ou plus) dans des domaines des sciences humaines (histoire, philosophie, sémiologie et littérature); en poste depuis plus de 10 ans; et, surtout, se connaissant les uns les autres et se positionnant les uns par rapport aux autres.

Dans le même temps, des différences au sein de ce pôle apparaissent, notamment lorsqu'il s'agit de situer les maisons d'édition en fonction du " degré de consécration ${ }^{24}$ ». La situation de chaque maison sur l'échelle de la consécration permet de distinguer deux groupes : le groupe des « avant-gardes consacrées ${ }^{25}$ » (ici Verticales et « Fictions \& Cie ») et celui des « tenants ${ }^{26}$ » du champ (ici les Éditions du Seuil, Gallimard et Flammarion). Ces deux groupes manifestent également leur cohésion en termes à la fois de caractéristiques sociales relatives au niveau de diplôme (les avant-gardes consacrées cumulent plus de diplômes que les tenants du champ) et de discours tenus (les avantgardes consacrées tiennent plus souvent et plus directement des discours distinctifs s'adressant à des éditeurs ou des maisons tenants du champ). 
Cet article s'appuie sur deux séries d'entretiens réalisés entre 2016 et 2018 avec des éditeurs parisiens appartenant à ce pôle de l'édition littéraire consacrée, que complètent des entretiens menés au cours de la même période avec des personnels de renfort œuvrant aux côtés des éditeurs (15 entretiens en tout). L'étude se fonde également sur une enquête ethnographique de trois mois effectuée au sein des Éditions du Seuil qui visait à saisir le travail des divers $\cdot e \cdot s$ agent $\cdot e \cdot s$ engagé $\cdot e \cdot s$ dans la publication d'un ouvrage. Par ailleurs, une recherche spécifique sur les Éditions Verticales a donné lieu à une exploitation des archives, à un comptage du catalogue et à la création d'une base de données rendant compte des caractéristiques sociales des auteur·e·s publiéee.s par la maison depuis sa date de création.

L'analyse qui suit propose d'exploiter les entretiens, thématisés en fonction des types de discours récurrents, et de les mettre en perspective par le biais d'observations ethnographiques, de comptages, de la consultation d'archives et d'entretiens avec les autres personnels de renfort pour mettre au jour de la manière dont l'ensemble des éditeurs du pôle de l'édition consacrée se donne à voir de façon invariable d'une part, et en fonction de la position relative de chaque éditeur à l'intérieur de ce pôle d'autre part.

\section{Les discours à l'épreuve des conditions d'accès au terrain}

\section{Le rôle des maillons invisibles de la chaîne de coopération}

La littérature consacrée à la difficulté d'accès à des terrains d'élite est assez abondante. Elle pointe tantôt le mépris d'un champ vis-à-vis du travail de recherche ${ }^{27}$, tantôt l'imposition de courtes durées d'entretien qui ne permettent pas au'à la chercheur-se de maitriser ce dernier ${ }^{28}$, tantôt encore l'embarras de " poser les questions qui fâchent » face à un « imposant ${ }^{29}$ » qui révèle l'écart entre les dispositions relatives de chaque partie prenante de l'entretien (et ici à la défaveur du·de la chercheur·se). Si ces contraintes ne sont pas propres au champ éditorial, elles constituent une partie des embûches dans l'entrée sur le terrain.

Plus spécifiquement, des chercheurs·ses sur l'édition ont souligné les obstacles inhérents à une recherche sur les éditeurs : imposition du lieu de 
l'entretien et du temps accordé30; "sens commun savant ${ }^{31}$ » imposé au·à la jeune chercheur'se; "loi du silence ${ }^{32} »$; problèmes d'accès à des données chiffrées ${ }^{33}$; refus d'entretien en raison d'un désaccord épistémologique ${ }^{34}$. Il va s'agir ici de cerner, à partir d'études de cas ou de récurrences qui paraissent particulièrement saillantes, voire généralisables, ce que les problèmes propres à l'entretien avec un éditeur, à plus forte raison appartenant à une maison consacrée, révèlent de l'identité que l'éditeur se construit et de ses effets sur le marché des biens symboliques.

Tout d'abord, et en dépit des conseils prodigués par Muriel Darmon ${ }^{35}$, la "présentation de $\operatorname{soi}^{36}$ » comme faisant partie du champ universitaire constitue une condition nécessaire mais non suffisante pour accéder à certains éditeurs. Il en va de même pour l'ajout d'une recommandation ("je me permets de venir de la part de...»), qu'elle soit éditoriale ou universitaire - à l'exception d'une recommandation provenant d'un'e auteur'e « connu'e » et «reconnu e » publié e par la maison d'édition en question. L'éditeur, probablement très sollicité d'une part, peut-être en quête d'une reconnaissance académique mais en même temps en proie à la méfiance d'autre part, ne répond pas au·à la chercheur·se inconnu'e qui n'a encore produit aucuns travaux et dont il ne connait pas les intentions. Il refuse même très vigoureusement de collaborer lorsque les postulats de recherche ne lui conviennent pas $^{37}$.

Extrait de mail à destination d'un éditeur de Flammarion

Bonjour Monsieur,

Je me permets de vous contacter sur conseils de [nom de l'informateur] des éditions du Seuil, qui m'a indiqué que vous pourriez être en mesure de m'aider.

Actuellement étudiante à l'École des Hautes Études en Sciences Sociales, je réalise un projet de thèse en sociologie de l'édition. Je m'intéresse particulièrement à la fabrique du catalogue et me questionne particulièrement sur la difficulté pour les femmes d'accéder à la publication.

Je réalise pour le moment des recherches documentaires et de terrain pour alimenter mes recherches. À ce titre, votre expérience chez Flammarion me paraît très intéressante en ce qu'elle pourrait me donner des pistes de réflexion d'une part et d'autre part me fournir des données concrètes sur votre travail au quotidien. 
Je vous joins par ailleurs mon CV pour que vous puissiez jeter un œil à mon parcours universitaire et professionnel.

Dans l'attente de votre réponse, je vous souhaite un très bon week-end.

Bien cordialement.

Réponse mail en provenance de l'éditeur de Flammarion

Chère Madame,

Je ne vois pas en quoi je pourrais vous aider. En vingt ans d'exercice, je n'ai jamais constaté de difficulté particulière pour les femmes d'accéder à la publication. Notre maison a longtemps été dirigée par une femme, les départements de littérature, du savoir sont dirigés par des femmes, nous avons de nombreuses femmes directrices littéraires ou éditoriales... Le sexe n'est pas un critère de sélection lorsque je choisis de soutenir un manuscrit. Bien à vous.

Aussi aura-t-il fallu trouver d'autres entrées, plus informelles, et qui attestent du puissant pouvoir de certains gatekeepers : assistant $\cdot e \cdot s$ d'édition, chargé·e·s des manuscrits, attaché $\cdot \mathrm{e} \cdot \mathrm{s}$ de presse ou stagiaires se sont révélé $\cdot \mathrm{e} \cdot \mathrm{s}$ d'une très grande efficacité pour parvenir aux éditeurs les plus difficiles d'accès. La quasi-totalité des entretiens (en dehors de ceux menés grâce à l'aide d'un·e auteur•e) ont été rendus possible par l'entremise d'un ou plusieurs de ces «maillons invisibles ${ }^{38}$ » de la « chaîne de coopération ${ }^{39}$ ».

C'est en effet dans le cadre d'une relation de confiance longuement cultivée auprès d'une assistante des Éditions du Seuil que j’ai pu bénéficier d'une entrée privilégiée dans cette maison d'édition, au sein de laquelle j'ai effectué une observation ethnographique de plusieurs mois.

J'ai ainsi remarqué (notamment lorsque je contactais directement certains éditeurs et que leurs assistantes me répondaient d'en passer d'abord par elles) que certains « maillons invisibles » de la « chaîne de coopération » disposaient d'un relatif pouvoir en ce qui concerne l'accord de « droit d'entrée » : ici, les assistantes ont souvent fait office de «gardiennes» du terrain, et je devais d'abord négocier avec elles ma présence sur les lieux et un entretien avec les éditeurs. Cela s'est reproduit à chaque négociation d'entretien (hormis les entretiens réalisés grâce à la recommandation d'auteurs de la maison). 
Extrait d'un e-mail envoyé par une assistante d'édition des Éditions du Seuil

Bonjour Lilas,

Pour ce qui est de vos recherches en édition contemporaine et votre intérêt pour notre collection nous sommes heureux de voir que de jeunes étudiants s'y intéressent! Je pense que vous pouvez demander un rendez-vous à [éditeur de «Fictions \& Cie »] pour dans le courant du mois de février [me donne son adresse mail]. Mais mettez-moi en copie si vous voulez que ça aboutisse.

Forte de prémisses de réflexivité quant à la dimension critique et peut-être malvenue de mon objet d'études dans un univers qui possède sa raison d'être dans le monopole de la production de savoirs littéraires et savants, mais aussi de ma prise de conscience d'une hiérarchie assez rigide qu'il convient de respecter au risque d'être disqualifiée, j'ai donc pris contact avec les enquêté·e's suivant·e's par le biais d'abord des assistantes d'édition, des chargé $\cdot e \cdot s$ de manuscrits ou des assistant $\cdot e \cdot s$ de presse, dont le rôle pourrait s'apparenter à celui d'un «intendant»: ils·elles détiennent le pouvoir de donner accès à l'éditeur et à la maison; gèrent les aspects invisibles et pratiques de la vie de l'éditeur et de la maison (calendriers éditoriaux, agendas des éditeurs ou des chargé·e·s de presse, réservation d'hôtels ou de restaurants, gestion des mails, gestion administrative dans son ensemble); mais, en dépit d'un lien hiérarchique avec l'éditeur (qu'il soit formalisé lorsqu'il s'agit de l'assistante attitrée d'un éditeur, ou symbolique lorsque conféré par l'appartenance à un " groupe de statut ${ }^{40}$ » prestigieux), ne bénéficient que peu souvent (et moyennant l'existence de capitaux préalables, notamment un «carnet d'adresses» important) d'une ascension hiérarchique (c'est-à-dire devenir éditeur·trice ou attaché·e de presse). Leur rôle «figé » de «petites mains ${ }^{41} »$ implique en effet des dispositions très lointaines de celles des éditeurs. Il est également curieux de remarquer que cette « intendance » relève de caractéristiques sociales semblables, notamment en termes de genre et de capital culturel et social ${ }^{42}$.

Éditeur chez Verticales : Et c'est vraiment un modèle, qui est un modèle dominant, dans ce milieu et... Nous par exemple on est très amis, c'est une ancienne stagiaire, [nom de l'intendante], qui est l'assistante de [l'éditeur de «Fictions \& Cie»], et en fait c'est très marrant, parce si vraiment on veut être honnêtes, elle fait 80 $\%$ du boulot. Et alors ce qui est intéressant si on rentre vraiment 
les mains dans le cambouis, [...] c'est que l'homme, c'est celui qui est l'homme de goût, l'honnête homme de goût, qui prend la décision esthétique générale, comme ça, et puis ensuite y'a un travail, qui est un travail très... Qui est de mettre les mains dans le cambouis du texte, le relire, prendre des notes sur le côté, $[\ldots]$ et qui est un travail de petites mains. Qui est subdivisé à l'assistante, et ensuite à la préparatrice de copie. Et on dit pas "préparateur de copie ", on dit "préparatrice de copie», parce que majoritairement c'est des femmes. Et dans beaucoup de maisons d'édition c'est la préparatrice de copie qui fait l'intégralité du travail éditorial [...]. Et ce qui est vachement intéressant, et avec des préparatrices aussi, qui font ce boulot, selon le fait qu'il y ait des éditeurs qui sont... Genre Philippe Sollers, les gens comme ça, donc uniquement des éditeurs de goût, qui savent même pas se servir d'un ordinateur et donc tout le reste c'est de l'intendance.

À l'instar des attachées de presse de l'édition étudiées par Delphine Naudier, voire à plus forte raison, les « intendantes » des maisons d'édition sont aussi essentielles qu'invisibilisées au profit de la « griffe ${ }^{43}$ » d'un grand nom : « Si la face émergée met en évidence des "griffes" (les noms des auteur·e·s, les éditeurs'trices) qui travaillent sur le matériau littéraire, les attachées de presse sont au contraire fondues dans le corps de leur fonction subordonnée ${ }^{44}$. En cela, les intendantes sont donc aisément remplaçables et leur statut s'oppose, de la même façon que celui des attachées de presse, à la personnalisation très forte des éditeurs qui, plus qu'une fonction, incarnent un nom.

De même, et d'une manière peut-être encore plus saillante, j'ai rencontré des difficultés (qui ont couru sur une année) pour obtenir un entretien avec le directeur de la collection « Fictions \& Cie » des Éditions du Seuil, alors même que j'étais très régulièrement présente sur les lieux. Souvent absent, j’ai dû compter sur son assistante pour pouvoir l'approcher - laquelle a fini par inscrire notre rendez-vous dans son agenda. Le jour du rendez-vous, je me suis présentée à l'accueil comme d'accoutumée. La secrétaire de l'accueil a alors tenté plusieurs fois de joindre l'éditeur au téléphone - sans succès. J'ai proposé de patienter dans le hall, craignant que le déplacement ne fût vain. S'est alors jouée une scène absolument extraordinaire de coopération entre les assistant $\cdot e \cdot s$ d'édition de la maison: une première assistante, que j'avais rencontrée lors d'un stage antérieur, m'a questionnée sur la raison de ma présence. À la suite de ma réponse, elle m'a ouvert le portique dont seule la 
possession d'un badge autorise le passage. Prenant de mes nouvelles, elle m'a conduite au bureau de l'éditeur en question - qui était bel et bien absent. J'ai choisi une nouvelle fois d'attendre. Patientant dans le couloir de son bureau, je croise une nouvelle assistante d'édition qui me questionne sur l'avancement de mes recherches. Je lui fais également part de ma mésaventure avec l'éditeur qui, depuis plus de 30 minutes, n’a toujours pas donné signe de vie. Cette deuxième assistante frappe au bureau d'une troisième, lui demandant où se trouve l'éditeur, laquelle parcourt le couloir pour aller à la rencontre d'une quatrième, qui m'indique enfin que l'éditeur se trouve à un autre étage. Elle propose de l'attendre avec moi. L'éditeur finit par faire son apparition dans le couloir au bout d'une heure et l'ensemble des assistantes mobilisées jusqu'alors le sollicitent une à une pour lui faire part de ma présence. Contraint par un couloir apparemment résolu à ce qu'il m'accorde un entretien, il m'invite à rejoindre son bureau (je réalise très rapidement qu'il avait oublié le rendez-vous et qu'il aura bien fallu la mobilisation de cinq assistantes en tout pour réussir à obtenir quelque 45 minutes d'interview).

\section{Négation du travail des intermédiaires au profit d'un éditeur omniscient et singulier}

Si ces conditions d'accès peuvent paraittre a priori anodines, elles ont un écho particulier lorsqu'on les met en résonance avec le discours que les éditeurs tiennent sur leur propre travail. En effet, en situation de "représentation », les éditeurs étudiés « cherche[nt] à donner une certaine image [d'eux-mêmes] » et, pour ce faire, opèrent un «tri entre ce que l'on dit et ce que l'on ne dit pas $^{45}$ ». Il s'avère que, sur l'ensemble des entretiens, tous les éditeurs ont souhaité être nommément cités; de la même façon, la totalité a signifié à au moins une reprise que je devais "absolument dire cela dans ma thèse », s'inquiétant parfois du fait que l'enregistreur puisse ne plus fonctionner. Une fois l'éditeur du pôle consacré atteint, celui-ci se donne à voir d'une façon particulière: il est un grand nom, et l'entièreté des publications de son catalogue ne relève que de lui. Figure de proue de la " chaîne de coopération ", visage public, personnalité singulière et singularisée dans nombre de travaux de recherche menés sur les éditeurs, l'éditeur du pôle étudié nie en général le travail collaboratif qu'implique l'édition d'un livre. 
Ainsi, à la suite des entretiens, j’ai thématisé les discours les plus récurrents des éditeurs du corpus. Tout d'abord, au regard des conditions d'accès aux éditeurs, j'ai cherché à déterminer s'il était fait mention des personnels de renfort et, si oui, dans quels termes. Il apparait que les personnels de renfort ne sont évoqués que par Verticales, spontanément et à quatre reprises lors de l'entretien. L'éditeur explique ainsi que le ou la stagiaire aide à opérer un tri des manuscrits et s'occupe de la communication de la maison sur internet (notamment sur les réseaux sociaux). Le ou la stagiaire n'est pas nommé $\cdot$, mais son travail est explicité et sa présence valorisée par l'éditeur : «Quand le ou la stagiaire a une sensibilité très littéraire, $[. .$.$] c'est vrai que c'est agréable$ aussi, parce que ça confronte des lectures générationnelles. » Il évoque également les deux lectrices extérieures qui participent à l'effort de sélection des manuscrits, laquelle aboutit à une «prise de décision collégiale ». Hormis cet éditeur, il n'est, dans tous les autres entretiens recensés, jamais spontanément question des personnels de renfort, à part lorsque je pose directement la question du rôle joué par ces derniers.

Ici, le travail des maillons invisibles est en grande partie éludé, principalement par les tenants du champ, au profit d'une personnalité qui garantit le cachet du livre publié et ainsi sa valeur sur le marché des biens symboliques. Aussi les éditeurs préfèrent-ils s'attacher plus longuement - et spontanément - à leur travail de « découvreurs » : «nous apportons des auteurs soit parce que nous les connaissons, soit parce qu'ils nous ont envoyé les manuscrits personnellement, soit même parce qu'on les a découverts dans tous les manuscrits qui arrivent », m’explique un éditeur du Seuil.

Ainsi, le travail en amont de l'édition d'un ouvrage n'entre pas en ligne de compte, alors que le travail de l'intendante revient souvent à dénicher les manuscrits arrivés par la poste et à les défendre auprès des éditeurs, comme en témoigne l'expérience d'une intendante aux Éditions du Seuil :

Question : Cette auteure, c'est aussi pour ça que tu l'as défendue, parce que c'est arrivé par la poste et que c'était quelque chose de bien... C'était un premier roman?

Intendante : Oui c'est son premier roman. [Elle] m'a envoyé son recueil de nouvelles et c'est comme ça que je l'ai repérée. Question : Et du coup ensuite toi t'en as parlé à [éditeur du Seuil]? Si on prend cet exemple. 
Intendante : Non je l'ai présenté en comité. Tout simplement. J'ai fait une fiche.

Question: Donc tu as porté ce projet comme éditrice... Finalement tu fais donc aussi un peu le travail d'un éditeur, non? Intendante : Ben à ce niveau-là oui.

Question : Et y'a beaucoup de gens qui l'ont lu ce roman?

Intendante : Deux éditeurs. [...] Un des éditeurs a trouvé que l'histoire était très forte, le sujet était vraiment intéressant, ouais c'était une très belle histoire. [...] Je pense qu'il voulait lui proposer de la rencontrer.

Question : Mais du coup il a repris la main sur le manuscrit?

Intendante : Oui, ben c'est moi qui suis allée le voir et qui lui ai demandé de le faire.

Question : D'accord. Pourquoi t'es allée le voir?

Intendante: Ben j'avais envie qu'on continue quand même à l'encourager, je pense que c'est un auteur. [...]

Question : Et toi tu n'aurais pas pu continuer à accompagner cette auteure que tu as découverte?

Intendante : Ben là de toute façon il faut qu'elle trouve un autre éditeur... Je vais l'aider peut-être à trouver un autre éditeur parce que je ne suis pas sûre que [l'éditeur] décide finalement de la publier.

Question : Ok. Non mais en fait ce qui me questionne c'est... Pourquoi est-ce que c'est lui qui...

Intendante : Ben lui il est éditeur, je ne suis pas éditrice.

Au-delà de la découverte des manuscrits, les éditeurs ne font pas non plus mention des acteurs trices qui interviennent dans le suivi du manuscrit (révisions, corrections, liens avec l'auteur·e à propos de la mise en livre, puis promotion de l'ouvrage avant et après sa parution). Jamais les éditeurs ne nomment les agent $\cdot e \cdot s$ en charge du «travail sur le texte » ou les personnes engagées dans la promotion du livre (à part dans le cas du ou de la stagiaire chez Verticales). Ces travailleurs'ses apparaissent simplement sous le couvert d'entités collectives ou de pronoms impersonnels chez Gallimard (« on »; « la maison») ou au Seuil («les attachées de presse »; «la famille Seuil»), ou n’apparaissent pas du tout pas du côté de « Fictions \& Cie ».

Si les personnes collaborant à la découverte, à la fabrication et à la promotion des ouvrages sont passées sous silence, l'éditeur fait a contrario part des grands noms qui ont accompagné sa carrière, rencontrés par «affinités littéraires » 
(Seuil), «atomes crochus » («Fictions \& Cie»), ou « hasard» (Gallimard), et qui semblent affermir la légitimité de sa position.

L'identité de l'éditeur, jalonnée par les grands noms de l'édition qui ont participé à sa construction, est affirmée par d'autres récurrences transversales de tous les entretiens: la défense de ses goûts littéraires singuliers; la cohérence de son catalogue, souvent à partir de la mention des auteur'e's qui ont fondé la carrière de l'éditeur (apparaissant ici comme "auteur d'auteur » pour reprendre l'expression employée par Régis Debray ${ }^{40}$ ); la personnalisation des rapports entretenus avec les auteur'e's; et enfin, le recours à des stratégies distinctives directement adressées aux éditeurs appartenant au sous-champ de l'édition consacrée, voire aux éditeurs appartenant à la même maison. En ce qui concerne ces stratégies, elles sont plus systématiquement usitées chez le pôle de l'avant-garde consacrée (Verticales et «Fictions \& Cie »).

S'agissant des goûts littéraires et de la composition du catalogue, ils se construisent autour des noms d'auteur'e's, et ce, dans tous les entretiens recensés. Ainsi, les catalogues sont décrits dans un aller-retour entre les prédilections préexistantes de l'éditeur (pour «le domaine de l'intime », ou " pour la littérature maghrébine, pour des raisons personnelles et familiales » aux Éditions du Seuil) et une catégorisation des auteur·e's qui permet à l'éditeur de généraliser ses préférences et de donner une homogénéité empirique à son catalogue (" prise en charge du sujet dans la narration chez Camille Laurens ou Christine Angot » chez Gallimard). Quoi qu'il en soit, les goûts de l'éditeur empruntent à la singularité de ses auteur·e·s, une singularité qui autorise néanmoins les rapprochements et, par-là, assure la cohérence et le prestige de son catalogue.

Par ailleurs, la singularité de la figure de l'éditeur est souvent renforcée par les rapports entretenus avec les auteur·e's : si les éditeurs ont parfois tendance à s'effacer devant « l'auteur charismatique ${ }^{47}$ », il n'en demeure pas moins qu'ils effectuent un « transfert de sacralité en ravissant aux premiers une part de leur magistère et de leur aura ${ }^{48}$ ». Ce transfert de sacralité s'opère bien plus facilement dans le cadre de relations personnelles et personnalisées qui mettent, un temps, l'auteur·e et son éditeur sur un pied d'égalité. Aussi l'édition est-elle « une histoire de relations » chez Gallimard; l'éditeur étant 
« tout le temps l'interlocuteur de l'auteur » au Seuil. Plus précisément, l'amitié est présentée comme étant au cœur des relations auteur·e-éditeur, ce dont témoignent de façon significative les entretiens réalisés au Seuil ou à Gallimard :

Éditeur du Seuil : Quand Nelly s'est suicidée, ce n'était que la énième tentative... Les autres tentatives $j$ 'en étais toujours prévenu dès le début, et avec le décalage horaire ça donnait des choses bizarres, je m'apprêtais à me coucher, on m'appelait... "Nelly a tenté de se suicider, elle est hospitalisée, on sait pas dans quel état elle est", je dormais plus de la nuit, à minuit j'avais un autre coup de fil "Non elle est consciente, elle est hors de danger, etc." Voilà, j'avais droit à des épisodes comme ça. C’est ça aussi être éditeur. Question : Donc vous aviez des liens forts avec...

Éditeur du Seuil : Ouais moi très forts avec elle et elle très forts avec moi parce que j'étais un peu sa bouée.

Éditeur chez Gallimard : Camille Laurens c'est une personne que j'aime bien. Et donc ça rend mon travail facile. C'est une personne auprès de qui j'apprends énormément. Parler de littérature avec Camille c'est passionnant. Mais sur un plan humain et professionnel c'est super pour moi. Elle connait mes enfants, je connais les siens, je m'intéresse à ce qu'ils deviennent, elle s'intéresse toujours à ce que deviennent les miens. C'est une vraie relation pleine entre éditeur et auteur.

Enfin, le recours à des stratégies distinctives caractérise le pôle de l'avantgarde consacrée: l'identité du pôle de l'avant-garde se bâtit donc contre l'identité des éditeurs les plus consacrés du champ. Ainsi, là où le discours des éditeurs de Verticales défend un mode d'organisation et un rapport au texte très différents des maisons qu'ils qualifient d'« aristocratiques », l'éditeur de « Fictions \& Cie » entame l'entretien avec une anecdote significative en termes de distinction (et revient, par ailleurs, sans cesse à une opposition entre les choix de la collection et la direction des Éditions du Seuil) :

Éditeur de «Fictions \& Cie » : Donc [Denis Roche] propose aux éditions du Seuil de lancer une nouvelle collection, il fait un petit projet, il vient l'exposer au grand comité du Seuil, grand comité c'est-à-dire qui a réuni l'ensemble des départements, sciences humaines, littérature, littérature étrangère etc., et Paul Flamand, qui est à l'époque encore PDG du Seuil, fait un tour de table et 
après l'exposé de Denis Roche, tout le monde se prononce contre la création de cette collection. Et Paul Flamand a ce mot magnifique, il dit « eh bien, puisque tout le monde est contre, on va la faire».

Aussi les stratégies d'approches déployées au cours de la recherche deviennent-elles de véritables outils heuristiques qui éclairent d'un jour nouveau les discours que l'éditeur tient sur lui-même, à savoir qu'il est un personnage solitaire et omniscient dans le processus de production et de promotion littéraire; mais également un personnage singulier dans ses goûts, et grâce aux relations personnelles qu'il entretient avec les auteur'e's de son catalogue. Mis en lien avec les observations de terrain, ces discours ne révèlent qu'une partie de la réalité du travail éditorial - éludant, en effet, l'implication des personnels de renfort.

Par ailleurs, si l'on observe plus finement les discours tenus par chaque éditeur en fonction de sa position dans le champ littéraire, leur uniformité est à nuancer légèrement, en ce que les stratégies distinctives se déploient davantage dans les discours des avant-gardes consacrées d'une part, et que, d'autre part, Verticales est la seule maison à faire mention des personnels de renfort qui participent à la production éditoriale.

Au-delà de l'analyse discursive, il est également apparu que Verticales se montrait plus facile d'accès que les éditeurs ayant acquis une position dominante dans le champ; les éditeurs de Verticales étant probablement conscients de la nécessité de bâtir une «domination traditionnelle ${ }^{49}$ » désormais détenue par les maisons d'édition les plus installées du champ - qui s'avèrent aussi les plus étudiées ${ }^{50}$.

L'étude approfondie de ces avant-gardes, qui m’ont rapidement donné un accès privilégié à leurs discours d'une part et à leurs archives d'autre part, m'a permis d'interroger un autre aspect du discours éditorial : celui, à la frontière de l'éditorial et du scientifique, de la maitrise de l'histoire de la maison. 


\section{La présentation de soi à l'épreuve des archives et du comptage systématique du catalogue}

\section{Discours linéaire et histoire maîtrisée}

En ce qui concerne tout d'abord leur discours, il s'est avéré très long, réflexif et surtout distinctif vis-à-vis des maisons d'édition dominantes dans le champ :

Éditeur chez Verticales : On ne veut pas faire comme les grandes maisons installées qui ont complètement reproduit un modèle très ancien et qui est un domaine de domination [...] sociale, masculine, tous les auteurs doivent être nés à Paris, dans certains arrondissements, avoir fait des études.... Y'a toujours ce modèle de l'écrivain normalien, brillant, tête bien faite, avec de temps en temps une femme qui est comme ça aussi, mais... C'est un modèle qui est encore très très dominant dans les grandes maisons parisiennes. [...] Mais c'est vrai que pour nous... y compris aussi dans les manuscrits, les manuscrits qu'on a pu accepter, les premiers romans, qu'on a souvent reçus par la poste, bon ben y'a une majorité de provinciaux, et de gens qui n'ont pas fait Normale, et pis de gens qui peuvent être au chômage ou au RSA.

En même temps, l'entretien s'est révélé, comme à chaque fois, très linéaire lorsqu'il s'agissait de penser l'histoire de la maison. L'éditrice de Verticales, m'expliquant que la maison est « rôdée » concernant son histoire, entame alors une description des tournants de la maison mentionnant des dates précises («1997, date à laquelle Bernard a décidé de fonder la maison », «En 1998, ils ont été deux », "Fin 1999, Slatkine a décidé de revendre la maison », «Fin 1999, le Seuil a racheté la maison », «Entre 2000 et 2004, on a décidé de faire beaucoup de premiers romans »), ou encore des affiliations éditoriales spécifiques («Bernard Wallet» est évoqué 18 fois dans l'entretien), et des noms d'auteur'e's qui ont permis d'élargir le catalogue de la maison ( Le catalogue s'est étoffé avec Pierre Senges, François Bégaudeau, y’avait déjà Olivia Rosenthal en 1999, mais se sont ajoutés donc Pierre Senges, Bégaudeau, Maylis de Kerangal »).

Grâce à un accès inédit aux archives de la maison, j’ai pu mettre en perspective les éléments du discours de l'éditeur et de l'éditrice sur la maison avec des 
éléments dépouillés dans les archives (contrats d'auteur·e·s, échanges entre auteur'e's et éditeurs, archives de presse, bilans comptables principalement). Par ailleurs, l'éditeur et l'éditrice m'ayant confié l'ensemble de leur catalogue, j’ai pu réaliser une base de données recensant l'ensemble du catalogue des origines de la maison à nos jours. À ce propos, l'éditeur se présentait comme l'héritier d'une maison fondée en 1997 par un éminent personnage du milieu littéraire parisien. En ce sens, la ligne éditoriale s'était affinée, mais n'avait guère bougé. Face à mes questionnements relatifs à leur rachat par le groupe Gallimard, ou aux éventuelles inflexions dans les politiques éditoriales du fait d'un marché de plus en plus compétitif, l'éditeur défendait une position de pourfendeur et de résistant. J'ai dû, pour passer outre ces discours, déconstruire l'histoire de la maison produite par les actuels éditeur et éditrice.

\section{Une sociologie de la production historique pour mettre au jour les enjeux du discours éditorial}

Afin de proposer une histoire de la maison rendant compte des évolutions à la fois en termes de politiques éditoriales, d'organisation du travail, de changements de maison-mère et d'acquisition de capitaux économiques et symboliques, j’ai tout d'abord souhaité déconstruire la chronologie proposée par Verticales sur son site internet. La chronologie organisée par rattachement à une maison-mère qu'établit la maison semble assez pertinente pour les deux premières périodes (période de rattachement à Slatkine et période de rattachement au Seuil), puisqu'elle correspond alors aux évolutions du catalogue et de l'organisation du travail (la période « Slatkine », entre 1997 et 1999, est marquée par un recrutement d'auteur'e's proches de Bernard Wallet, au profil plutôt masculin, et par une organisation du travail sur le modèle des maisons d'édition indépendantes, sur fond de grandes difficultés financières; la période de rattachement au Seuil marque au contraire l'acquisition d'une stabilité organisationnelle et financière et une expansion du catalogue à la faveur des premiers romans, en même temps que l'entrée de la maison dans la course aux prix et l'accès aux "poche » à travers la filiale « Points » des Éditions du Seuil). Cependant, pour la période de rattachement au groupe Gallimard, l'évolution des politiques éditoriales et du capital symbolique ne coïncide pas entièrement avec la période présentée par l'éditeur et l'éditrice (2006-2017). Si ces derniers proposent la scission à partir du rachat par le groupe Gallimard, il s'avère que le véritable tournant de la 
politique éditoriale de la maison se trouve plutôt aux alentours de la période 2009-2010, qui marque en fait le départ de Bernard Wallet des Éditions Verticales. En effet, à partir de cette date, le nombre de publications est divisé par 1,7 (passant à 9,75 titres par an en moyenne contre 16,5 titres par an en moyenne sur la période précédente), le nombre de premiers romans publiés est divisé par 3,5 (3,4 par an en moyenne pour la période 2000-2009 à moins de 1 à partir de 2009), et, surtout, l'obtention de prix littéraires par des auteur'e's de la maison s'accroît nettement et de façon durable. Ainsi, à partir de 2009, quelque 26 prix sont décernés aux auteur·e's de la maison, dont le premier grand prix d'automne en 2010. La moyenne annuelle d'un prix avant le départ de Bernard Wallet s'élève à 3,6 dès le départ du fondateur. La maison recentre également son catalogue autour du roman contemporain et évacue peu à peu les genres littéraires les moins rentables (essais, reportages, poésie, théâtre...). Pour cette déconstruction/reconstruction de l'histoire de la maison, j'ai établi une frise chronologique à plusieurs étages illustrant diverses évolutions thématisées ("rattachement à un groupe ", "évolutions du catalogue » (densité, diversité, profil sociologique des auteur·e·s), « organisation du travail », " prises de position dans la presse »). J'ai également produit une base de données mettant au jour l'évolution des caractéristiques sociales des auteur·e·s publié $\cdot e \cdot s$. Cette étude du développement de la maison en fonction de son organisation, de son rattachement à un groupe, de ses prises de positions et de la composition de son catalogue m'a permis de distinguer les moments de rupture, de proposer de nouvelles bornes temporelles répondant à d'autres logiques et de pointer l'impact du départ du fondateur dans le recentrement du catalogue sur la fiction française contemporaine. Ce recentrement a conduit à une moindre diversité sociale des auteur·e·s publié e $\cdot s$. Ainsi, le départ du fondateur de la maison a été omis pour penser le bornage chronologique, probablement par souci de proposer une histoire de la continuité (les références à Bernard Wallet sont, comme dit, nombreuses dans les entretiens réalisés avec les actuels éditeur et éditrice) plutôt que de la rupture - or il s'agit d'un tournant significatif en termes de politiques éditoriales et qui a affecté durablement la structure et la densité du catalogue des éditions.

Cela m'a valu une virulente dispute avec l'éditeur et l'éditrice de Verticales, et la fermeture d'une partie de mon terrain. Pour autant, en analysant avec plus de recul la situation dans laquelle nous nous sommes trouvés en 
confrontation, j'ai saisi ce que mon «faux pas » révélait des discours de ces éditeurs, et probablement plus largement des éditeurs appartenant au pôle de l'avant-garde consacrée : ces derniers ont remarquablement conscience de la nécessité de produire leur propre histoire, héritée de grands noms dont la légitimité ne serait pas remise en cause. En ce sens, la maison s'investit particulièrement sur internet pour donner accès à ses archives, retracer son histoire, et elle a récemment créé sa page Wikipédia. En d'autres termes, les éditeurs étudiés, très lucides quant à la valeur d'une histoire maittrisée, exercent et continuent d'exercer un monopole important sur leur propre histoire, duquel il est très difficile de se déprendre.

Le constat d'une histoire maîtrisée n'est pas à imputer simplement aux avantgardes les moins consacrées du champ. Cette stratégie a en effet été mise en œuvre chez «Fictions \& Cie» et chez Gallimard, repérable à travers la mention de dates précises qui bornent l'histoire de la maison, de la collection ou du catalogue; visible également lorsque l'éditeur revendique un héritage éditorial particulier (dans le cas de «Fictions \& Cie », l'éditeur cite Denis Roche dès le début de l'entretien, et revient sur le parcours du fondateur en employant le temps présent : «Denis Roche, il est formé par les avant-gardes. Très très jeune, il est proche des surréalistes $[. .$.$] et très vite il va rejoindre le$ groupe Tel Quel, dont il va finir par se séparer »), ou encore son appartenance à l'histoire longue d'un courant littéraire. Ainsi, chez Gallimard, l'éditeur d'une partie des œuvres de Christine Angot et de Camille Laurens m'explique prendre en charge une partie de l'héritage autofictionnel des années 1980 et contribuer à sa légitimation et son renouvellement. Dans le même temps, il insiste sur la véracité de ce discours, à la façon d'un historien : «Alors je vais vous répondre précisément en vous donnant les faits de manière chronologique », précisant qu'il "préfère donner des résultats bons plutôt que des résultats dont [il n’est] pas certain ».

La thématique d'une histoire linéaire, renforcée par des affiliations éditoriales et des dates-clés destinées à mettre en valeur la maison, contribue à conférer à l'éditeur une place de choix dans le champ littéraire, comme le rappelle à juste titre Hervé Serry à propos des Éditions du Seuil : «Le passé, sans cesse interprété et réinterprété [...], est une ressource déterminante pour l'avenir de toute entreprise d'édition ${ }^{51}$. » Par les discours qu'il tient sur lui-même et sur son travail, l'éditeur du pôle consacré se donne à voir comme un acteur 
singulier et omniscient dans le processus de publication. Les conditions d'accès à ses discours mais aussi les témoignages des personnels de renfort permettent autant d'interroger la représentation qu'il se fait de lui-même que de relativiser sa place dans la chaine éditoriale. Lorsque l'on prend en compte la position relative de chaque éditeur dans le champ, il s'avère toutefois que les «avant-gardes» les moins consacrées (ici l'éditeur et l'éditrice de Verticales) se distinguent par leur reconnaissance du travail des personnels de renfort. Aussi les autoreprésentations de l'éditeur en situation d'entretien sont-elles également à évaluer en fonction de son degré de consécration.

Cette étude a permis de rendre compte des principaux aspects qui gouvernent les discours de l'éditeur appartenant au pôle de l'édition consacrée (omniscience, singularité et maîtrise de l'histoire héritée), en même temps que de proposer au’à la chercheur'se des outils (observation ethnographique, comptage, analyse du discours des gatekeepers, recours aux archives) favorisant tant la distinction de ce qui relève proprement du « discours » que l'analyse, au plus près du terrain, du travail éditorial réel d'une part, et des stratégies de domination éditoriale d'autre part.

Quoi qu'il en soit, les discours tenus par les éditeurs appartenant au pôle le plus consacré de l'édition française semblent avoir pour principale fonction de garantir la valeur symbolique des ouvrages publiés. Aussi l'omission du travail collectif et la maitrise d'une histoire prestigieuse confèrent-elles à ces éditeurs une aura symbolique particulièrement solide sur la scène nationale (Paris demeurant assurément la capitale éditoriale, notamment dans l'espace francophone, comme le rappelle Josée Vincent ${ }^{52}$ ) et internationale ${ }^{53}$. Ils contribuent donc, par la « croyance $^{54}$ » en leur propre singularité et à travers les modalités de leur construction, à assurer à une partie de la littérature française contemporaine une valeur symbolique à peu d'autres égale.

Plus largement, il parait légitime de se demander si la recrudescence des travaux et témoignages sur les éditeurs et l'édition depuis les années 1980, dans un contexte de rationalisation éditoriale ${ }^{55}$ et de globalisation ${ }^{56}$ qui tend à réduire l'intérêt des ouvrages à des enjeux strictement financiers ${ }^{57}$, n'est pas sans lien avec un discours performatif de résistance éditoriale passant par le canal d'une histoire linéaire des grands noms et favorisant, du moins en France, une relative perpétuation de la croyance en la valeur des livres publiés. 
Lilas Bass est doctorante à l'École des Hautes Études en Sciences Sociales (EHESS) sous la direction de Gisèle Sapiro (CNRS-EHESS) et chercheuse invitée de la Bibliothèque nationale de France. Elle mène une thèse sur les conditions de publication, de publicisation et de commercialisation des écrivaines au sein des maisons d'édition en littérature française contemporaine (de 1980 à nos jours). Elle participe par ailleurs à une enquête collective sur les agents littéraires internationaux, coordonnée par Tristan Leperlier et Gisèle Sapiro.

\section{Notes}

1 Sylvie Noël, L'édition indépendante critique : engagements politiques et intellectuels, Villeurbanne, Presses de l'Enssib, 2012, p. 34; voir également : Michel Pinçon et Monique Pinçon-Charlot, Voyage en grande bourgeoisie : journal d'enquête, Paris, Presses universitaires de France, 1998.

2 Pierre Bourdieu, Le sens pratique, Paris, Minuit, 1980, p. 117.

3 Stéphane Beaud et Florence Weber, Guide de l'enquête de terrain. Produire et analyser des données ethnographiques, Paris, La Découverte, 2010, p. 126.

${ }^{4}$ Sur l'utilité d'un entretien « raté », voir : Muriel Darmon, «Le psychiatre, la sociologue et la boulangère : analyse d'un refus de terrain ", Genèses, n 1, 2005, pp. 98-112.

${ }^{5}$ Hélène Chamborédon et al., «S'imposer aux imposants. À propos de quelques obstacles rencontrés par des sociologues débutants dans la pratique et l'usage de l'entretien », Genèses, vol. 16, no 1, 1994, pp. 114-132.

${ }^{6}$ Christelle Avril, Marie Cartier et Delphine Serre, Enquêter sur le travail. Concepts, méthodes, récits, Paris, La Découverte, 2010, p. 43.

7 Stéphane Beaud et Florence Weber, "Observer», Guide de l'enquête de terrain. Produire et analyser des données ethnographiques, Paris, La Découverte, 2010, pp. 125-153.

8 Au sens donné par Bertrand Legendre, c'est-à-dire dans la perspective de donner à voir une image particulière de soi-même; Bertrand Legendre, "Introduction", dans Bertrand Legendre et Christian Robin (dir.), Figures de l'éditeur, Paris, Nouveau Monde éditions, 2005, p. 27.

9 Jérôme Meizoz, Postures littéraires. Mises en scène modernes de l'auteur, Genève, Slatkine, 2007.

10 Pierre Bourdieu, Les règles de l'art. Genèse et structure du champ littéraire, Paris, Seuil, coll. « Points », 1998 [1992].

${ }^{11}$ Howard Saul Becker, Les mondes de l'art, Paris, Flammarion, 1988.

Vol. 10, $\mathrm{n}^{\circ} 2$ | Printemps 2019

«Les discours de l'éditeur » 
${ }^{12}$ Notamment : Pierre Bourdieu, Les règles de l'art. Genèse et structure du champ littéraire, Paris, Seuil, coll. «Points », 1998 [1992].

${ }^{13}$ Comme le recommande vivement Anthony Glinoer dans sa recension de Wenceslas Lizé, Delphine Naudier et Olivier Roueff, Intermédiaires du travail artistique. À la frontière de l'art et $d u$ commerce (Ministère de la Culture et de la Communication, Département des études, de la prospective et des statistiques, coll. «Questions de culture », 2011), Sociologie de l'Art, vol. 3, opus 21, 2012, pp. 143-148.

${ }^{14}$ Sophie Noël, «Maintenir l'économie à distance dans l'univers des biens symboliques : le cas de l'édition indépendante "critique" ", Revue française de socio-économie, vol. 2, n' 10, 2012, pp. 73-92.

15 Pierre Bourdieu, «La production de la croyance : contribution à une économie des biens symboliques ", Actes de la recherche en sciences sociales, vol. 13, n 1, 1997, pp. 3-43.

${ }^{16}$ Howard S. Becker, Les mondes de l'art, Paris, Flammarion, 1988.

17 Marie-Christine Bureau et Emmanuelle Marchal, «Pluralité des marchés du travail et qualités des intermédiaires », document de travail du Centre d'étude de l'emploi, nº 48, 2005.

18 Wenceslas Lizé, Delphine Naudier et Olivier Roueff, Intermédiaires du travail artistique. À la frontière de l'art et du commerce, Ministère de la Culture et de la Communication, Département des études, de la prospective et des statistiques, coll. «Questions de culture », 2011, p. 15.

19 Pierre Bourdieu, «La production de la croyance : contribution à une économie des biens symboliques ", Actes de la recherche en sciences sociales, vol. 13, n 1, 1997, pp. 3-43.

20 Christophe Charle, "Le temps des hommes doubles», Revue d'histoire moderne et contemporaine, tome 39, $\mathrm{n}^{\circ}$ 1, janvier-mars 1992, pp. 73-85.

21 Pierre Bourdieu, «Le champ littéraire », Actes de la recherche en sciences sociales, vol. 89, septembre 1991, pp. 3-46, p. 7.

22 Pierre Bourdieu, «La production de la croyance : contribution à une économie des biens symboliques », Actes de la recherche en sciences sociales, vol. 13, nº 1, 1997, p. 12.

23 Pierre Bourdieu, "Le champ littéraire », Actes de la recherche en sciences sociales, vol. 89, septembre 1991, p. 16.

${ }^{24}$ Pierre Bourdieu, Les règles de l'art. Genèse et structure du champ littéraire, Paris, Seuil, coll. « Points », 1998 [1992], p. 205.

25 Pierre Bourdieu, Les règles de l'art. Genèse et structure du champ littéraire, Paris, Seuil, coll. « Points », 1998 [1992], pp. 205-208 : certaines avant-gardes littéraires, pour faire date, sont contraintes de se plier, dans leurs propositions formelles et leur mode de fonctionnement, aux exigences de la «consécration bourgeoise» (p. 208). À ce titre, elles renoncent partiellement à leurs propositions avant-gardistes en même temps qu'elles accèdent aux prix, à un plus large lectorat et à la possibilité d'entrer dans l'histoire.

Vol. 10, $\mathrm{n}^{\circ} 2 \mid$ Printemps 2019

«Les discours de l'éditeur» 
${ }^{26}$ Pierre Bourdieu, « La production de la croyance : contribution à une économie des biens symboliques ", Actes de la recherche en sciences sociales, vol. 13, n 1, 1997, p. 31.

${ }^{27}$ Muriel Darmon, «Le psychiatre, la sociologue et la boulangère : analyse d'un refus de terrain », Genèses, n 1, 2005, pp. 98-112.

${ }^{28}$ Stéphane Beaud, «L'usage de l'entretien en sciences sociales. Plaidoyer pour l'“entretien ethnographique" », Politix, vol. 9, n 35, 1996, pp. 226-257, p. 238.

${ }^{29}$ Sylvain Laurens, " "Pourquoi” et "comment" poser les questions qui fâchent? Réflexions sur les dilemmes récurrents que posent les entretiens avec des "imposants" ", Genèses, vol. 4, n 69,2007 , pp. 112-127.

${ }^{30}$ Sophie Noël, L'édition indépendante critique : engagements politiques et intellectuels, Villeurbanne, Presses de l'Enssib, 2012, p. 36.

31 Patrick Champagne, «La rupture avec les préconstructions spontanées ou savantes », dans Patrick Champagne, Rémi Lenoir, Louis Pinto et Dominique Merllié, Initiation à la pratique sociologique, Paris, Dunod, 1989, pp. 163-220.

32 Hervé Haraon et Patrick Rotman, Les intellocrates. Expédition en haute intelligentsia, Paris, Ramsay, 1981, p. 144.

33 Paul Dirkx, «Les obstacles à la recherche sur les stratégies éditoriales », Actes de la recherche en sciences sociales, vol. 126-127, mars 1999, pp. 70-74, p. 71.

${ }^{34}$ Bruno Auerbach, «L'édition en sciences sociales : une approche socio-épistémologique. Productions et marchés; évaluation et sélection; mise en texte et mise en livre », thèse de doctorat, Paris, École des Hautes Études en Sciences Sociales, 2006, p. 482.

35 Muriel Darmon, «Le psychiatre, la sociologue et la boulangère : analyse d'un refus de terrain », Genèses, n 1, 2005.

${ }^{36}$ Erving Goffman, La mise en scène de la vie quotidienne. 1. La présentation de soi, Paris, Minuit, coll. « Le Sens commun », 1973.

${ }^{37}$ Comme déjà relevé en annexe de la thèse de Bruno Auerbach («L'édition en sciences sociales : une approche socio-épistémologique. Productions et marchés; évaluation et sélection; mise en texte et mise en livre », thèse de doctorat, Paris, École des Hautes Études en Sciences Sociales, 2006), mais guère analysé plus avant.

38 Delphine Naudier, "Les attachées de presse : les maillons invisibles de l'édition », dans Delphine Naudier, Catherine Marry et Marie Buscatto (dir.), Actes de la journée d'études "Travail, genre et art », Paris, Document de travail du Mage, 13, pp. 35-46.

${ }^{39}$ Howard S. Becker, Les mondes de l'art, Paris, Flammarion, 1988.

${ }^{40}$ Concept wébérien de « ständische Lage », ordinairement traduit par « groupe de statut » mais également par « conditions », qui prend en considération « un privilège positif ou négatif de considération sociale »; Max Weber, Économie et société 1. Les catégories de la sociologie, Pocket, coll.

Vol. 10, $\mathrm{n}^{\circ} 2 \mid$ Printemps 2019

«Les discours de l'éditeur» 
«Agora», 1995 [1971], p. 395. Autrement dit, l'expression wébérienne permet de complexifier l'analyse de la stratification sociale en introduisant la question du prestige tiré « [du] mode de vie, de la naissance, ou de la profession » (p. 396).

${ }^{41}$ Pour reprendre les termes de l'éditeur de Verticales.

${ }^{42}$ L'ensemble des intendantes qui m'ont ouvert l'accès au terrain avaient les caractéristiques sociales suivantes : femmes, 35 ans ou plus, niveau BAC +5 ou plus, diplômées de grandes écoles (Sciences Po ou École normale supérieure - donc, surqualifiées pour la plupart des tâches requises dans leur quotidien), nées et/ou ayant étudié à Paris, possédant un capital social faible, ayant un salaire moyen autour de 2000 euros nets par mois.

43 Pierre Bourdieu, «Le couturier et sa griffe : contribution à une théorie de la magie », Actes de la recherche en sciences sociales, vol. 1, $\mathrm{n}^{\circ}$ 1, 1975, pp. 7-36.

${ }^{44}$ Delphine Naudier, «Les attachées de presse : les maillons invisibles de l'édition », dans Delphine Naudier, Catherine Marry et Marie Buscatto (dir.), Actes de la journée d'études "Travail, genre et art», Paris, Document de travail du Mage, 13, p. 42.

45 Bertrand Legendre, «Introduction », dans Bertrand Legendre et Christian Robin (dir.), Figures de l'éditeur, Paris, Nouveau Monde éditions, 2005, pp. 27-28.

${ }^{46}$ Expression utilisée par Anne Simonin, « Le catalogue de l'éditeur, un outil pour l'histoire. L'exemple des Éditions de Minuit ", Vingtième Siècle. Revue d'histoire, vol. 1, no 81, pp. 119129, p. 122, et empruntée à Régis Debray, Le pouvoir intellectuel en France, Paris, Ramsay, 1979.

47 Marie-Pier Luneau, «De la culpabilité d'être marchand: duplicité de l'auteur-éditeur. L'exemple de Jacques Godbout », dans Bertrand Legendre et Christian Robin (dir.), Figures de l'éditeur, Paris, Nouveau Monde éditions, 2005, p. 60.

48 Jean-Yves Mollier, "Naissance de la figure de l'éditeur », dans Bertrand Legendre et Christian Robin (dir.), Figures de l'éditeur, Paris, Nouveau Monde éditions, 2005, p. 17.

${ }^{49}$ C'est-à-dire une forme de domination reposant sur «le caractère sacré de dispositions [Ordnungen] transmises par le temps ("existant depuis toujours") et des pouvoirs du chef » (Max Weber, Économie et société 1. Les catégories de la sociologie, Pocket, coll. «Agora », 1995 [1971], p. 302), chef(s) ou autorité(s) qui tirent ainsi leur légitimité de la transmission de règles et qui s'imposent dans le respect de la tradition. Pour ce qui nous intéresse ici, il s'agit de discriminer les maisons d'édition qui ont routinisé les modes d'organisation du travail, les valeurs de la maison, mais également les règles de passation du pouvoir grâce, notamment, à une histoire formelle (écrits, témoignages, études sur les maisons d'édition), des maisons d'édition dont l'histoire est plus récente et qui ne bénéficient pas de ces cadres routinisés d'action, d'interaction et d'imposition d'un pouvoir légitime au sein et en dehors de la maison.

${ }^{50}$ Sophie Noël, L'édition indépendante critique : engagements politiques et intellectuels, Villeurbanne, Presses de l'Enssib, 2012, p. 13.

Vol. 10, n 2 | Printemps 2019

«Les discours de l'éditeur » 
${ }^{51}$ Hervé Serry, «Figures d'éditeurs français après 1945 : habitus, habitus professionnel et transformation du champ éditorial », dans Bertrand Legendre et Christian Robin (dir.), Figures de l'éditeur, Paris, Nouveau Monde éditions, 2005, p. 72.

52 Josée Vincent, «Axes territoires. Introduction », dans Bertrand Legendre et Christian Robin (dir.), Figures de l'éditeur, Paris, Nouveau Monde éditions, 2005, p. 281.

${ }^{53}$ La France était en effet classée deuxième sur la liste des pays qui vendent le plus de droits étrangers en 2014; Étude du Motif, «Comment vendre des droits à l’étranger? », Paris, 2014, p. 8 .

${ }^{54}$ Pierre Bourdieu, «La production de la croyance : contribution à une économie des biens symboliques ", Actes de la recherche en sciences sociales, vol. 13, n 1, 1997.

55 À propos du phénomène de regroupement éditorial, voir notamment : Pierre Bourdieu, "Une révolution conservatrice dans l'édition ", Actes de la recherche en sciences sociales, vol. 126127, mars 1999, pp. 3-28; et John B. Thompson, Merchants of Culture, Cambridge, Polity Press, 2010.

${ }^{56}$ Consulter à ce propos : Gisèle Sapiro (dir.), Les contradictions de la globalisation éditoriale, Paris, Nouveau Monde, 2009.

${ }_{57}$ Philippe Bouquillion et al., «À propos des mouvements récents (2004- 2005) de concentration capitalistique dans les industries culturelles et médiatiques ", Le Temps des médias, vol. 1, nº 6, 2006, pp. 151-164.

\section{Bibliographie}

Bruno Auerbach, «L'édition en sciences sociales : une approche socioépistémologique. Productions et marchés; évaluation et sélection; mise en texte et mise en livre ", thèse de doctorat sous la direction de Jean-Louis Fabiani, Paris, École des Hautes Études en Sciences Sociales, 2006.

Christelle Avril, Marie Cartier et Delphine Serre, Enquêter sur le travail: concepts, méthodes, récits, Paris, La Découverte, 2010.

Stéphane Beaud, "L'usage de l'entretien en sciences sociales. Plaidoyer pour 1'“entretien ethnographique" ", Politix, vol. 9, n 35, troisième trimestre 1996, pp. 226-257.

Stéphane Beaud et Florence Weber, Guide de l'enquête de terrain. Produire et analyser des données ethnographiques, Paris, La Découverte, 2010.

Howard Saul Becker, Les mondes de l'art, Paris, Flammarion, 1988.

Marie-Christine Bureau et Emmanuelle Marchal, «Pluralité des marchés du travail et qualités des intermédiaires », document de travail du Centre d'étude de l'emploi, $n^{\circ} 48,2005$.

Vol. 10, $\mathrm{n}^{\circ} 2 \mid$ Printemps 2019

« Les discours de l'éditeur» 
Philippe Bouquillion et al., « À propos des mouvements récents (2004-2005) de concentration capitalistique dans les industries culturelles et médiatiques », Le Temps des médias, vol. 1, n 6, 2006, pp. 151-164.

Pierre Bourdieu, «Le couturier et sa griffe : contribution à une théorie de la magie », Actes de la recherche en sciences sociales, vol. 1, n 1, 1975, pp. 7-36.

Pierre Bourdieu, «La production de la croyance : contribution à une économie des biens symboliques ", Actes de la recherche en sciences sociales, vol. 13, n 1, 1977, pp. 3-43.

Pierre Bourdieu, Le sens pratique, Paris, Minuit, 1980.

Pierre Bourdieu, Les règles de l’art, Paris, Seuil, coll. «Points essais », 1998 [1992].

Pierre Bourdieu, «Une révolution conservatrice dans l'édition », Actes de la recherche en sciences sociales, vol. 126-127, mars 1999, pp. 3-28.

Hélène Chamborédon et al., «S’imposer aux imposants. À propos de quelques obstacles rencontrés par des sociologues débutants dans la pratique et l'usage de l'entretien », Genèses, vol. 16, n 1, 1994, pp. 114-132.

Patrick Champagne, «La rupture avec les préconstructions spontanées ou savantes ", dans Patrick Champagne, Rémi Lenoir, Louis Pinto et Dominique Merllié, Initiation à la pratique sociologique, Paris, Dunod, 1989, pp. 163-220.

Christophe Charle, "Le temps des hommes doubles », Revue d'histoire moderne et contemporaine, tome 39, n 1, janvier-mars 1992, pp. 73-85.

Muriel Darmon, «Le psychiatre, la sociologue et la boulangère : analyse d'un refus de terrain », Genèses, no 1, 2005, pp. 98-112.

Paul Dirkx, "Les obstacles à la recherche sur les stratégies éditoriales », Actes de la recherche en sciences sociales, vol. 126-127, mars 1999, pp. 70-74.

Anthony Glinoer, "Wenceslas Lizé, Delphine Naudier et Olivier Roueff, Intermédiaires du travail artistique. À la frontière de l'art et du commerce, Ministère de la Culture et de la Communication, Département des études, de la prospective et des statistiques, coll. «Questions de culture », 2011 », Sociologie de l'Art, vol. 3, opus 21, 2012, pp. 143-148.

Erving Goffman, La mise en scène de la vie quotidienne. 1. La présentation de soi, Paris, Minuit, coll. « Le Sens commun », 1973.

Hervé Haraon et Patrick Rotman, Les intellocrates. Expédition en haute intelligentsia, Paris, Ramsay, 1981.

Vol. 10, n 2 | Printemps 2019

« Les discours de l'éditeur» 
Sylvain Laurens, "Pourquoi" et "comment" poser les questions qui fâchent? Réflexions sur les dilemmes récurrents que posent les entretiens avec des "imposants" ", Genèses, vol. 4, n 69, 2007, pp. 112-127.

Bertrand Legendre et Christian Robin (dir.), Figures de l'éditeur, Paris, Nouveau Monde éditions, 2005.

Wenceslas Lizé, Delphine Naudier et Olivier Roueff, Intermédiaires du travail artistique. À la frontière de l'art et du commerce, Paris, Ministère de la Culture et de la Communication, Département des études, de la prospective et des statistiques, coll. «Questions de culture », 2011, pp. 25-27.

Jérôme Meizoz, Postures littéraires. Mises en scène modernes de l'auteur, Genève, Slatkine, 2007.

Motif, « Comment vendre des droits à l'étranger? », Paris, 2014.

Delphine Naudier, «Les attachées de presse : les maillons invisibles de l'édition », dans Delphine Naudier, Catherine Marry et Marie Buscatto (dir.), Actes de la journée d'études "Travail, genre et art », Paris, Document de travail du Mage, $\mathrm{n}^{\circ}$ 13, 2008, pp. 35-46.

Sylvie Noël, L'édition indépendante critique: engagements politiques et intellectuels, Villeurbanne, Presses de l'Enssib, 2012.

Sophie Noël, "Maintenir l'économie à distance dans l'univers des biens symboliques : le cas de l'édition indépendante "critique" ", Revue française de socioéconomie, vol. 2, n 10, 2012, pp. 73-92.

Michel Pinçon et Monique Pinçon-Charlot, Voyage en grande bourgeoisie: journal d'enquête, Paris, Presses universitaires de France, 1998.

Gisèle Sapiro (dir.), Les contradictions de la globalisation éditoriale, Paris, Nouveau Monde, 2009.

Anne Simonin, «Le catalogue de l'éditeur, un outil pour l'histoire : l'exemple des Éditions de Minuit », Vingtième Siècle. Revue d'histoire, vol. 81, no 1, 2004, pp. 119-129.

John B. Thompson, Merchants of Culture, Cambridge, Polity Press, 2010.

Max Weber, Économie et société 1. Les catégories de la sociologie, Paris, Agora, coll. « Pocket», 1995 [1922]. 